\title{
Adaptation to frequency-shifted auditory feedback
}

\author{
STUART M. ANSTIS \\ York University, Downsview, Ontario, Canada \\ and \\ PATRICK CAVANAGH \\ Université de Montréal, Montreal, Quebec, Canada
}

\begin{abstract}
Subjects attempted to whistle at the frequency of a target note when the sound of their own whistling, fed back through headphones, was either electronically shifted in frequency or blotted out by intense masking noise. With auditory feedback masked out, they could still hit a note to within .5-2 semitones, so proprioception from lips and tongue must be internally calibrated to about this accuracy, in the absence of auditory feedback. When the frequency of their whistling was electronically shifted by plus or minus $300 \mathrm{~Hz}$, they adjusted the frequency they produced, shifting it down or up just enough to hold constant the frequency feedback to their ears. Thus, auditory feedback is used to fine-tune the motor output, and overrides it when a cue conflict is experimentally introduced. When the electronic frequency shift was gradually increased from zero to $\pm 300 \mathrm{~Hz}$, subjects adapted to the shift, and when they subsequently tried to hit a target note with auditory feedback masked off, they showed a negative aftereffect of $50 \%-60 \%$, making frequency errors of about \pm 150 $180 \mathrm{~Hz}$. Finally, after adapting to a shift of $\pm 250 \mathrm{~Hz}$, subjects attempted to reset the electronic frequency shift to subjective zero and again showed aftereffects of $45 \%-77 \%$. Proprioceptive and auditory feedback information in controlling whistling can be compared with proprioceptive and visual feedback in controlling limb position.
\end{abstract}

How are we able to sing or whistle in tune? Two different kinds of information are available for controlling the frequency (pitch) of sung or whistled notes: proprioceptive information about the conformation of lips and tongue, and auditory feedback of the actual note produced. There is anecdotal evidence that both kinds of information are used to control speech, singing, etc. Auditory feedback is important: deaf people of ten talk too loudly, and so does a man temporarily deafened by an electric razor held close to his face and ears. Delayed auditory feedback disrupts speech severely (Chase, Sutton, \& First, 1959; Katz \& Lackner, 1977). On the other hand, auditory feedback is not essential, since totally deaf people can learn to talk quite effectively. Also, a trained singer seems to be able to hit a desired note instantly, without the need (or the time) to sing a trial note and then adjust its pitch by means of auditory feedback. Presumably the proprioceptive information is calibrated internally so that both deaf and normal people know that a particular conformation of the vocal tract will produce a desired sound.

We gratefully acknowledge receipt of Grants AO260 to S.M.A. and A8606 to J.P.C., both from the National Science and Engineering Research Council of Canada (NSERC). We thank Dr. Brian Rogers for his invaluable help in running the experiments.
In this study, we investigated the role of proprioceptive information vs. auditory feedback in controlling the frequency (pitch) of whistling. We found that the motor output was easily and rapidly recalibrated neurally when the auditory feedback was electronically shifted in frequency. Subjects were asked to whistle in tune with a target tone. In our first experiment, auditory feedback was blotted out by very loud masking noise. Result: Subjects could still come within .5-1 semitone of a target tone despite their inability to hear themselves. In our other experiments, the auditory feedback, delivered through headphones, was electronically shifted up or down in frequency before the subjects heard it. In this cue conflict situation, subjects responded according to the auditory feedback and ignored proprioceptive cues. Furthermore, subjects adapted to this frequency shift in the feedback. When feedback was subsequently blotted out in test trials, they showed negative aftereffects, making frequency errors in their whistling which were opposite in sign and about half the magnitude of the electronic frequency shift.

We studied whistling rather than singing for technical, not logical, reasons. Pilot work reminded us that one can hear one's own voice via bone conduction through the skull. No tolerable level of masking noise could really guarantee to block out 
this unwanted bone conduction, since even the faintest auditory feedback of the voice through the skull could defeat the purpose of our Experiment 1. Whistling produces much less conduction through the skull than does singing, since singing involves vibrations of the larynx which are transmitted through the skull, whereas whistling vibrates a column of air whose acoustic impedance is too low to set the skull vibrating. This difference in bone conduction can be demonstrated by blocking one ear with a finger, which favors skull conduction over ossicle conduction. Singing or humming will sound much louder in the blocked ear, as every chorister knows. On the other hand, whistling will sound only marginally louder in the blocked ear.

Whistling does seem to give good proprioceptive cues. The reader can verify for himself that as he whistles up the scale he will feel his tongue moving forwards and upwards in the mouth. Oddly enough, the fact that the larynx changes its position as one sings up the scale is often not realized by a naive singer until he first rests his fingers on his Adam's apple. For the purposes of this paper, we use the word "proprioceptive" loosely, lumping together motor outflow, efferent copy, sense of effort, etc., together with sensory inflow from the lips and tongue.

\section{EXPERIMENT 1: WHISTLING WITHOUT AUDITORY FEEDBACK}

Subjects heard, through headphones, a series of random target tones from a signal generator, and were asked to whistle at the frequency of each tone when they heard it.

The frequencies of the target tone and of the whistled response were displayed on a Fluke 1900 frequency meter, out of the subject's sight, and were written down by the experimenter. The meter's resolution was set to the nearest $10 \mathrm{~Hz}$ : It was found impracticable to read it to greater accuracy, owing to fluctuations over time in the meter's righthand digit, caused by fluctuations in the subject's whistling. The whistled frequencies were also converted into ratios, by dividing each one by the target frequency. Thus, if the target tone was $1,500 \mathrm{~Hz}$ and the subject whistled at $1,700 \mathrm{~Hz}$, the ratio was $1700 / 1500=1.1333$. In this example, he would be whistling sharp by $13.33 \%$, or just over 2 semitones. One semitone on the even-tempered scale is equal to $5.95 \%$.

\section{Control Condition: Whistling with Feedback}

Subjects heard each tone and tried to whistle in tune with it. They could hear their own whistling undistorted through the headphones, and were free to use any beat frequency cues. Results for one subject (P.C.) are shown in Figure 1a. His performance was good; the standard deviation of his frequency ratios, which was taken as a measure of his random error, was equal to $1.13 \%$, or about .19 semitone.

\section{Whistling Without Auditory Feedback}

Target tones of random frequency were again presented in random order, but now intense masking white noise was added through the headphones. The target tone was loud enough to be heard through the noise, but it was ensured that the subject could no longer hear his own whistling, which was now switched off and not fed back. He was instructed to whistle softly to ensure that he did not inadvertently hear himself. Thus he was forced to rely on proprioceptive cues only. Results are shown for the two subjects in Figures $1 b$ and 1c. It will be seen that the ability to whistle in tune was reduced but not destroyed. Regression lines were fitted to the data. For P.C., the correlation coefficient $r$ was .996 , the slope of the best fitting line was 1.0004 , and the random error (SD) was $2.4 \%$, or .41 semitone. S.M.A.'s performance was not so precise. Here $r=.964$, the slope of the line was .87 , and the random error (SD) was $6.1 \%$, or 1.03 semitones.

As stated earlier, we used whistling rather than singing to reduce the risk of the subject's inadvertently hearing his own voice by air conduction rather than through the headphones. However, in pilot work we did find that singing with and without feedback gave results similar to whistling. A naive female student was presented with random target tones over a similar range of frequencies, and asked to sing (not whistle) at the target frequencies. Given full auditory feedback, her results gave a correlation coefficient (r) of .996, a slope of 1.037, and a random error (SD) of $2.1 \%$, or .35 semitone. With feedback blotted out by noise, $\mathrm{r}$ became .927 , the slope was .74 , and the random error (SD) rose to $11.0 \%$, or $1.85 \mathrm{semi}$ tones.

When auditory feedback is cut off, the subject is obliged to rely on open-loop control from proprioceptive/motor cues. The precision attained here, with standard deviations of a semitone or so, suggest that these proprioceptive cues must be neurally calibrated to a precision of a semitone or two.

Certainly, the removal of auditory feedback degraded the subjects' performance. The quality of their whistling, which they could not hear, often became breathy and tentative. And to a musician, errors of one or two semitones sound disastrous. But to a control engineer they would suggest that a considerable degree of control, albeit crude, is still being exercised by the proprioceptive information. Furthermore, these results set a lower bound upon the precision of the internal calibration, but not an upper one. A trained musician might do better than our relatively unmusical subjects. 

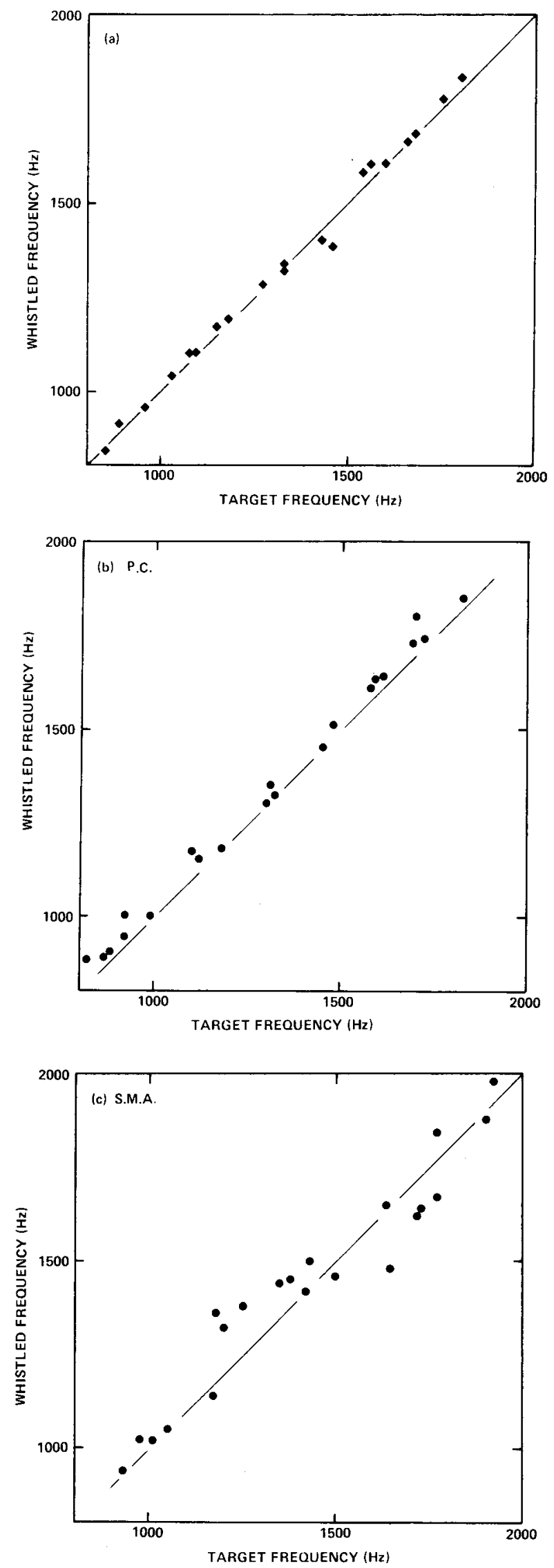

\section{EXPERIMENT 2: FREQUENCY-SHIFTED AUDITORY FEEDBACK}

In Experiment 1, adding auditory feedback in the control condition reduced the errors occurring from proprioceptive cues by a factor of 5 or 10 . It seems that the feedback provides fine tuning which overrides or corrects any inaccuracies in the proprioceptive system. In Experiment 2, we electronically shifted the frequency of the auditory feedback which the subject received through the headphones. Thus, proprioceptive cues signaled that the mouth was ready to deliver one note, but the feedback deceptively signaled that it had actually delivered a different note. In this experimental cue conflict, we found that subjects no longer whistled on the target tone: instead, they shifted the frequency at which they actually whistled by just enough to make the feedback frequency match the target tone. This shows that they controlled the frequency of their whistling by ignoring proprioceptive cues and attending exclusively to the auditory feedback.

\section{Method}

The subject's whistle was picked up by a microphone, amplified, and fed into the headphones via a Surrey Electronics ${ }^{1}$ Spectrum Shifter (Hartley-Jones, 1973). This device generates a shift frequency, $f_{s}$, from an internal oscillator, and electronically adds it to or subtracts it from any sinusoidal input frequency, $\mathrm{f}_{\mathrm{i}}$, which in this case was the subject's whistling. The frequency shifter's output was a sine wave of frequency $f_{i}+f_{s}$ (or $f_{i}-$ $f_{s}$ ), and this was fed to the subject's headphones. The target tone was always $1,220 \mathrm{~Hz}$. Four runs of 10 trials each were run. The frequency shift was zero on the first trial, and then gradually increased by $10 \mathrm{~Hz}$ on each trial, to a maximum of $+100 \mathrm{~Hz}$ on one run. On a second run, the frequency shift was downwards, taking the values $-10,-20,-30, \ldots,-90,-100 \mathrm{~Hz}$ on the 10 trials. On a third run, the frequency shift was incremented by $30 \mathrm{~Hz}$ on each trial: $0,+30,+60,+90 \ldots,+270,+300 \mathrm{~Hz}$. On a fourth run, the frequency shifts were downwards: $0,-30$, $-60,-90, \ldots,-270,-300 \mathrm{~Hz}$. The runs were presented in a different order to each of the three subjects. Shifts of \pm 100 and $\pm 300 \mathrm{~Hz}$ correspond to rises or falls in pitch of about 1.4 and 4.2 semitones.

\section{Results}

If a person controlled his whistling entirely by monitoring his own proprioceptive information, he would always whistle at a constant $1,220 \mathrm{~Hz}$, regardless of any frequency shift in the auditory feedback. If he relied entirely on auditory input, he would alter the frequency at which he whistled to compensate for shifts in feedback frequency in such a way as to hold constant the frequency of the auditory feedback $\left(f_{i} \pm\right.$ $f_{s}$ ). Thus, if the feedback was shifted upwards by

Figure 1.(a) Whistling with auditory feedback (control condition) was very accurate, with random error (SD) of only $1.13 \%$, or 19 semitone. (b) When feedback was masked out, performance fell off, but was still quite good under open-loop proprioceptive control. Random error (SD) was $2.4 \%$, or .4 semitone, for P.C. and $6.1 \%$, or 1.03 semitones, for S.M.A. 


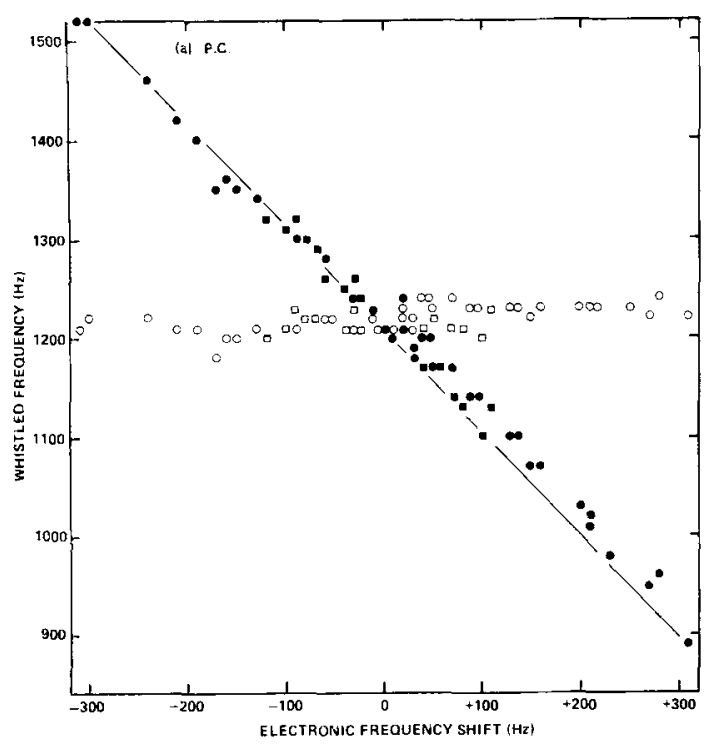

$+100 \mathrm{~Hz}$, he would be expected to shift his whistled tone downwards from 1,220 to $1,120 \mathrm{~Hz}$, thereby keeping the tone fed back to the headphones at a constant $1,220 \mathrm{~Hz}$.

The results clearly support the second hypothesis. Figure 2 shows that as the frequency shift inserted into the feedback loop was experimentally raised (or lowered), the subjects responded by lowering (or raising) the frequency of their whistling just enough to keep the frequency fed back to their ears equal to the target frequency of $1,220 \mathrm{~Hz}$. Thus, subjects acted to keep constant the frequency which they heard (after shifting), but they adjusted the frequency they produced by an amount equal and opposite to the electronic shift in the feedback loop. This is shown in Figure 2, where the (shifted) auditory feedback frequencies, shown as open symbols, lie along the horizontal line at about $1,220 \mathrm{~Hz}$, i.e., the fed back frequency was held constant. The frequencies of the actual whistles produced are shown as solid symbols. These are fitted by lines sloping downwards at $45^{\circ}$, i.e., the subjects lowered their output frequency to compensate when the experimenter raised the electronic shift frequency.

These results were not artifacts of the incremental method of gradually increasing the frequency shift. Comparable results were obtained (not shown here) when the frequency shift was randomly assigned on different trials to values of $-200,-100,-50,0$, +100 , and $+200 \mathrm{~Hz}$.

\section{EXPERIMENT 3: ADAPTATION TO SHIFTED AUDITORY FEEDBACK}

In this experiment, the feedback frequency shift was gradually increased on successive trials, as described above. Subjects compensated for the shift, and adapted to it, showing a negative aftereffect on subsequent test trials during which auditory feedback was blotted out.

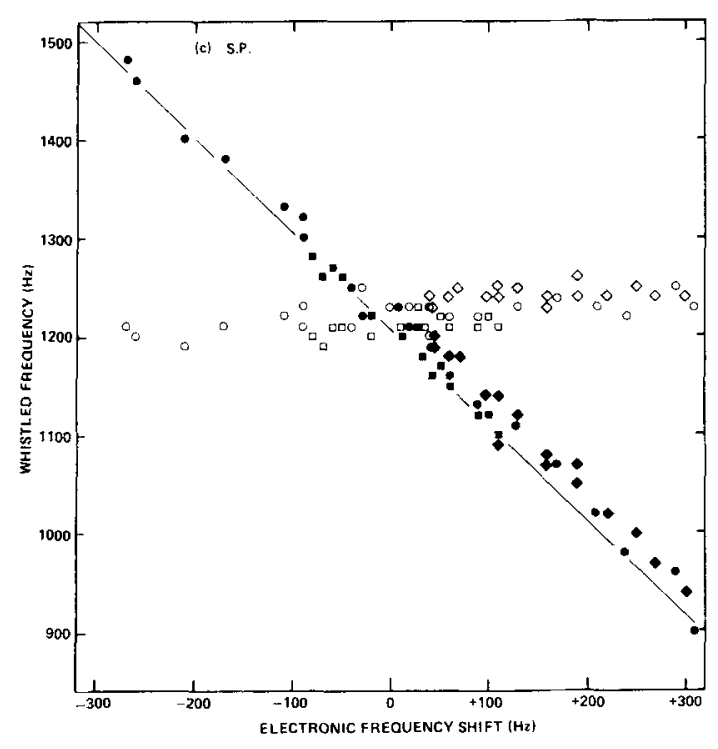

\section{Method}

Four conditions were run:

Condition 1: Control. Subjects made 10 attempts to whistle at the target frequency of $1,220 \mathrm{~Hz}$, with full undistorted feedback through the headphones.

Condition 2: Noise masked out feedback. Subjects made 10 further attempts to whistle at the target tone frequency of $1,220 \mathrm{~Hz}$ but this time intense masking noise prevented them from hearing their own whistles, which were now not fed back through the headphones.

Figure 2. When the fedback frequency was electronically shifted by various amounts, subjects adjusted their whistled outputs (- B) to hold the shifted frequency fed back to their ears ( $O \square$ ) at a constant $1,220 \mathrm{~Hz}$, equal to the target frequency. This suggests that subjects controlled whistling frequency by attending to auditory feedback, not proprioceptive cues. Different symbols indicate different runs. 
These two conditions replicated Experiment 1, except that only a single target tone of $1,220 \mathrm{~Hz}$ was now used instead of the previous range of target frequencies.

Condition 3: Adaptation to frequency-shifted feedback. This procedure has already been described under Experiment 2. Over a series of 10 trials, the feedback frequency shift was increased in steps of $+10 \mathrm{~Hz}$ to a final shift of $+100 \mathrm{~Hz}$, or decreased in steps of $-10 \mathrm{~Hz}$ to a final shift of $-100 \mathrm{~Hz}$, or increased in steps of $+30 \mathrm{~Hz}$ to a final value of $+300 \mathrm{~Hz}$, or decreased in steps of $-30 \mathrm{~Hz}$ to a final value of $-300 \mathrm{~Hz}$. On each trial, the subject attempted to whistle at the target frequency of $1,220 \mathrm{~Hz}$.

Condition 4: Test for adaptation. Immediately after Condition 3, the subjects made 10 attempts to whistle at the frequency of the $1,220-\mathrm{Hz}$ target tone, with auditory feedback masked out by intense noise, exactly as in Condition 2 . If the subject had adapted to the electronic frequency shifts imposed during Condition 3 , then the frequency of his whistling output would be different in Condition 4 (after adaptation) compared with Condition 2 (before adaptation).

\section{Results}

Results are illustrated in Figures 3 and 4. Figure 3 shows the mean and standard deviations of the whistling frequencies produced during each condition. In the baseline Condition 1 , the median standard deviation was $7 \mathrm{~Hz}$, or .1 semitone. In Condition 3, the subject raised or lowered the pitch of his whistling as the amount of feedback shift was varied, but he held very constant the frequency which was fed back to his ears. The median standard deviation was now $9 \mathrm{~Hz}$, or .13 semitone-hardly any larger than in Condition 1. Notice that the subject held this feedback frequency constant by making large compensatory changes in his actual whistled output, to match and null out the electronic frequency shift. The imposed shift did not significantly increase his random error. This indicated that he relied entirely on auditory feedback to steer his whistled frequencies: if he had attended to proprioceptive cues, this would have produced a considerable cue conflict, which would presumably have increased his random errors.

In Condition 2, when feedback was masked out, the median standard deviation rose to $37 \mathrm{~Hz}$, or .5 semitone. As in Experiment 1, the removal of auditory feedback degraded performance but did not destroy it. Presumably the neural calibration of proprioceptive information of mouth position is accurate to within half a semitone.

The most interesting part of Figure 3 is the responses to Condition 4. These showed clear evidence of adaptation to the electronic frequency shift. For example, when the feedback shift for subject S.M.A. was gradually lowered in Condition 3 to a final value of $-300 \mathrm{~Hz}$, he increased his whistling frequency by $+300 \mathrm{~Hz}$ to $1,520 \mathrm{~Hz}$, to hold his feedback frequency constant at the target frequency of $1,220 \mathrm{~Hz}$. Immediately afterwards, Condition 4 was presented, in which he made 10 attempts to whistle at the target frequency with his feedback masked out by noise. If he showed no adaptation, his whistling would revert straight back to $1,220 \mathrm{~Hz}$. If he showed $100 \%$ adaptation, he would continue whistling at $1,520 \mathrm{~Hz}$, even though he now received no frequency-shifted
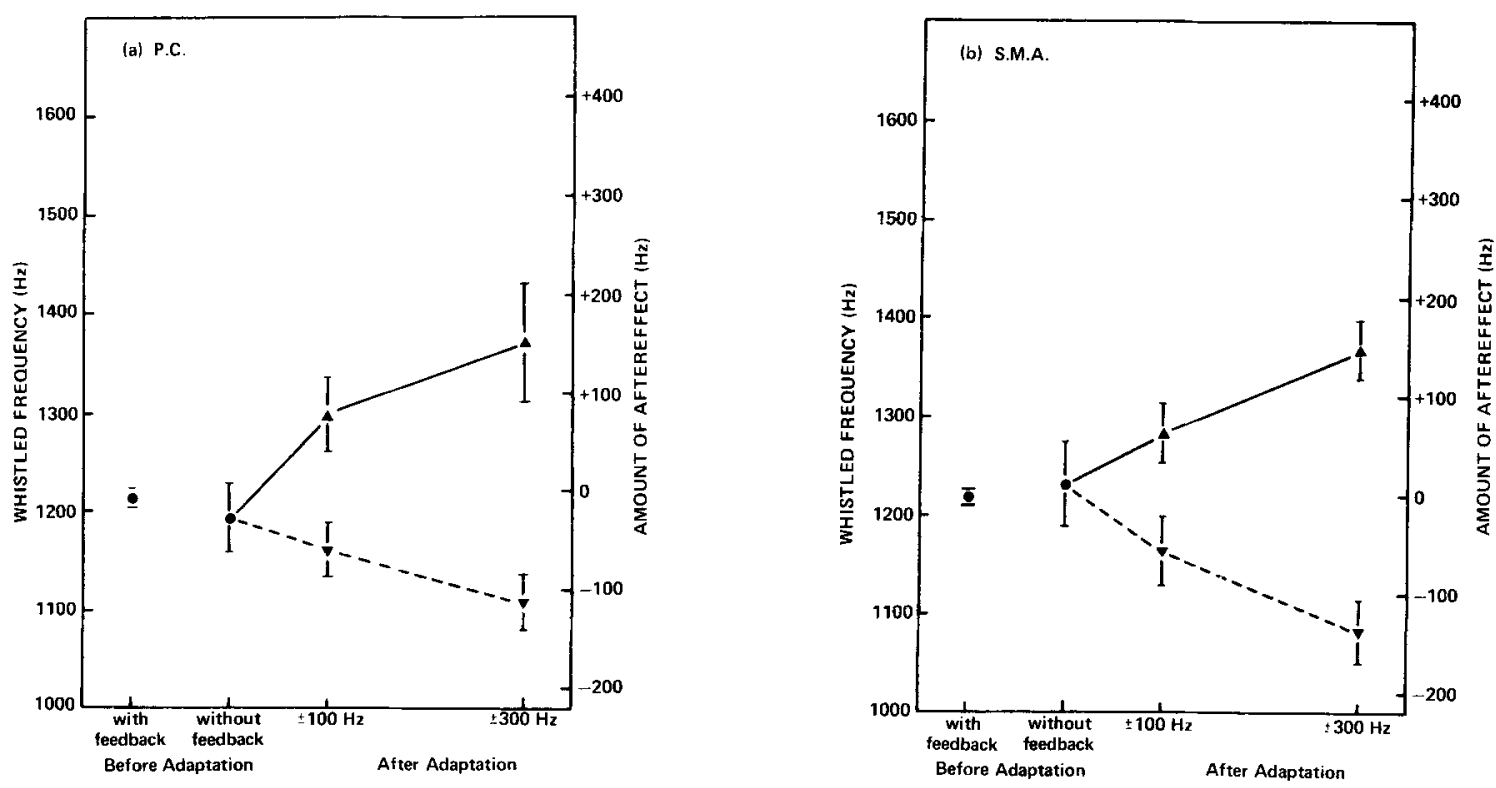

Figure 3. Adaptation to frequency-shifted auditory feedback. Before adaptation, subjects given feedback accurately reproduced the target frequency of $1,220 \mathrm{~Hz}$ with SDs of only 7-10 Hz (Condition 1), and without feedback (Condition 2) SDs rose to about $40 \mathrm{~Hz}$. During Condition 3 (not shown), subjects adapted to a frequency shift which was gradually increased to a final value of $-300,-100,+100$, or $+300 \mathrm{~Hz}$. In the test condition, No. 4, subjects showed af tereffects, whistling above the target frequency ( $\Delta$ ) after adapting to a downward electronic shift and whistling below the target frequency $(\nabla)$ after adapting to an upward shift. These aftereffects were $50 \%-60 \%$ of the adapting shift, and in the opposite direction to it. 
feedback, or, indeed, no feedioack of any kind. In fact, he whistled at about $1,400 \mathrm{~Hz}$-about $180 \mathrm{~Hz}$ above the target frequency. This represents an adaptation level of $60 \%(=180 / 300)$. Averaged over the four frequency shifts of \pm 100 and $\pm 300 \mathrm{~Hz}$, the mean level of adaptation was $53 \%$ for S.M.A. and $46 \%$ for P.C. The aftereffects failed to reach significance in two cases (S.M.A. adapting to $-100-\mathrm{Hz}$ shift, and P.C. adapting to $+100-\mathrm{Hz}$ shift), but the aftereffects in the other six conditions were significant at $p<.00001$ or even better.

The time courses of the adaptation to $\pm 300-\mathrm{Hz}$ frequency shifts are illustrated in Figure 4 . As the frequency shift was gradually raised (or lowered) on successive trials, the subjects gradually lowered (or raised) their whistling frequency, just enough to hold constant the frequency fed back to their ears. The filled symbols in Figure 4 show their gradually changing output whistles and the open symbols show the fed back frequencies. In the test condition No. 4, they maintained about $50 \%$ of this adaptive shift, showing an aftereffect in which they whistled about halfway between the adapting frequency and the target frequency.

\section{Control}

We suggest that the electronic shift in the feedback loop led to a recalibration of felt position of the lips and tongue. We ran a control condition to rule out the possibility that this recalibration was an open-loop aftereffect of "postural persistence" (Howard \& Anstis, 1974). For instance, if a blindfolded person holds both of his arms out horizontally in front of himself and then raises one arm $45 \mathrm{deg}$ above the horizontal for a few seconds, the arm is positioned above the other when the person is asked to restore the horizontal position. It might be that the mere act of whistling above (or below) the target frequency during the adapting trials would produce a postural persistence, leading to a kinaesthetic aftereffect of whistling above (or below) the target tone on test trials, completely independent of any auditory feedback.

To rule out this possibility, each subject whistled 10 times at a target tone of $1,520 \mathrm{~Hz}$ on one run and at $920 \mathrm{~Hz}$ on another run, with full undistorted auditory feedback through headphones. After each run, he made 10 attempts to whistle at a target tone of $1,220 \mathrm{~Hz}$ which he heard through headphones. Intense white noise masked out any auditory feedback. Note that if the aftereffect in the main experiment were merely postural, it would show up equally well in this control condition, but if it depended (as we believed) on auditory feedback, it would not show up. Result: No aftereffects were found in this control condition. We concluded that the aftereffects did depend on auditory feedback, and were not merely "postural persistence."
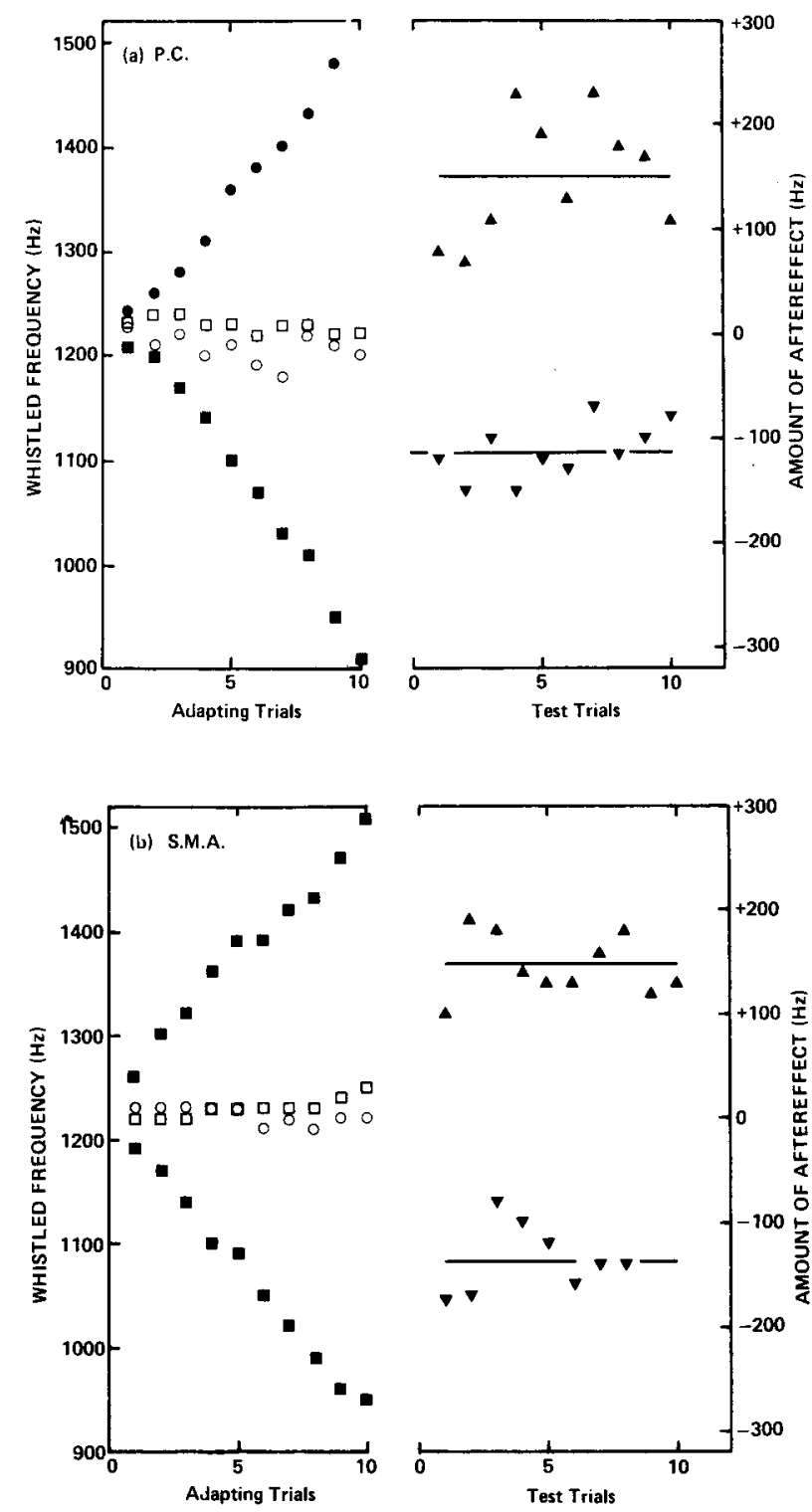

Figure 4. Time course of adaptation to electronic shifts of $\pm 300 \mathrm{~Hz}$. When the shift gradually fell to $-300 \mathrm{~Hz}$, the whistled output ( $\bullet$ ) rose to compensate, keeping the shifted tone fed back to the ears $(0)$ at a constant $1,220 \mathrm{~Hz}$. In the test condition, the aftereffect $(A)$ was about $50 \%-60 \%$ of the adaptation level. When the shift gradually rose to $+300 \mathrm{~Hz}$, the output whistle ( $\square$ ) fell, holding constant the fedback tone ( $\square$ ) and giving a corresponding aftereffect $(\nabla)$.

\section{EXPERIMENT 4: SUBJECTS RESET FEEDBACK FREQUENCY SHIFT TO APPARENT ZERO}

There is a possible objection to the experiments described so far: It might be that the subjects were merely making a conscious correction for the feedback frequency shifts. We need to show that the 
behavioral compensation was an essentially involuntary control of the subjects' behavior by the feedback, and not merely an intentional effort to stabilize the auditory feedback. Any such conscious, deliberate corrections would be like a simple learned skill overlying an unadapted perceptual system, and they would fail to show a true adaptive recalibration.

To overcome this objection, three modifications were made: (1) The apparatus was improved and automated, so that the frequency shift was gradually incremented electronically instead of manually during the course of the experiment. (2) Several of the subjects were experimentally naive. (3) The subjects' task was changed. After a gradual, progressive adaptation (as before), they were given a knob which controlled the amount of frequency shift in their feedback, and asked to set this fedback frequency shift to subjective zero.

If the subjects in the previous experiments had been merely making conscious corrections, then they would still have known at what pitch they were really whistling, so in this new experiment they would be expected to set the frequency shift veridically to zero. Thus, in the test condition, they would make settings which would allow them to hear their own whistles directly without any shift. But if the previous experiment had produced a true adaptation and recalibration, then in the present experiment they ought to reset the shift (mistakenly) to a nonzero value, which ought to be in the same direction as the adapting frequency shift. This offsetting of subjective zero by the subjects would represent a negative aftereffect. In fact, we did find such aftereffects, which could be up to $83 \%$ of the final adapting shift. We conclude that the adaptation and aftereffects are true recalibrations and can in no way be dismissed as mere conscious corrections made by the subjects.

\section{Method and Procedure}

The procedure was generally similar to that of Experiment 3, in that the subject adapted to a gradually increasing frequency shift over a series of trials. The shift was either upwards or downwards on different runs. As before, we found that the subject increased the actual pitch of his whistling if the frequency shift was downwards, and decreased it if the shift was upwards, always in such a way as to hold virtually constant the feedback frequency that he heard through the headphones. After the adapting period, his aftereffect was measured by a reset method and found to be substantial.

In the previous experiments, the frequency shift was manually set by the experimenter: the subject made a whistle, and the experimenter manually increased the frequency shift slightly, ready for the next trial. In the new setup, the frequency shift started at zero, and was then gradually and continuously increased, in either a positive or a negative direction (shift up or shift down), by means of a single, very slow electronic ramp, which lasted between 2 and $8 \mathrm{~min}$. This ramp was fed into the voltage-controlled frequency input of a generator which determined the amount of frequency shift. The target tone of $1,250 \mathrm{~Hz}$ sounded for a .5 -sec burst every $2.5 \mathrm{sec}$, allowing $2 \mathrm{sec}$ of silence in which the subject tried to whistle at the target frequency. At the beginning of the adapting period, the subject heard his own whistle through the headphones at its true frequency, i.e., with zero shift. But over the ensuing 2 min the fedback frequency gradually altered, reaching a final shift of $+250 \mathrm{~Hz}$ on half the trials and $-250 \mathrm{~Hz}$ on the other half. Result: His whistles gradually shifted up (for a downward frequency shift) or down (for an upward frequency shift), just enough to hold virtually constant the frequency fedback to his headphones. This behavior during adaptation was similar to that in the previous experiments.

The procedure to test for an aftereffect was quite different from before. During the test period, the target tone was turned off. The subject was asked to continue whistling at about the same frequency, and to adjust the electronic frequency shift back to zero by means of a control knob. Thus, he attempted to match the note he heard through the headphones to the true note that he was whistling. This was done 10 times in succession before adaptation to establish a baseline, and then again 10 times after the adapting period to measure any aftereffect, with 10 -sec readaptation periods between resettings. After he had reset the amount of shift to his own satisfaction as being subjective zero, he was readapted for $10 \mathrm{sec}$. During each readaptation period, he whistled to match four target tone bursts ( 4 bursts of $2.5 \mathrm{sec}=10 \mathrm{sec}$ ), with the electronic shift set to zero in the baseline condition and to +250 or $-250 \mathrm{~Hz}$ in the postadaptation condition. The experimenter then randomly offset the knob, and the subject made another setting.

The frequency shifts during adaptation were purposely incremented at such a slow rate the subject was unaware of the progressive change. The shift incremented gradually over at least a 2-min period, with a target tone and a whistled response every $2 . \overline{5} \mathrm{sec}$. This gave about 48 whistles during an adapting run, so the frequency shift altered by no more than $5 \mathrm{~Hz}(=250 / 48)$ on successive trials. Since the JND for frequency under optimal conditions is $3-4 \mathrm{~Hz}$ for a target tone of $1,250 \mathrm{~Hz}$, we felt that the $5-\mathrm{Hz}$ shift in these suboptimal conditions would be below threshold, as desired. And, in practice, the subjects were generally unaware of the directions of the gradually adapting shift, although they could learn to cheat a little on upward shifts, because they had to generate a very low-pitched whistle by the end of the run which "felt funny."

\section{Results}

During the adapting period, as the frequency shift was gradually increased from zero in an upward (downward) direction, the subjects decreased (increased) the pitch of their whistling just enough to hold virtually constant the frequency fed back to their ears (Figures 5a and 5b). This result confirms those of the previous experiments. Moreover, when questioned afterwards, naive subjects often reported that they had been maintaining a constant pitch output, and were very surprised on being told differently. We conclude that they were not merely making a conscious decision to hold the feedback constant by varying their whistling pitch. Rather, the shifted feedback was creating an involuntary change in the relationship between the position of lips/tongue and the pitch which the subject expected to produce.

During the test period, the subjects showed substantial aftereffects, offsetting the apparent zerofrequency shift in the same direction as the adaptingfrequency shift. Figure 6 shows data for all five subjects. In the baseline condition (preadaptation), settings of the frequency shift did not differ significantly 

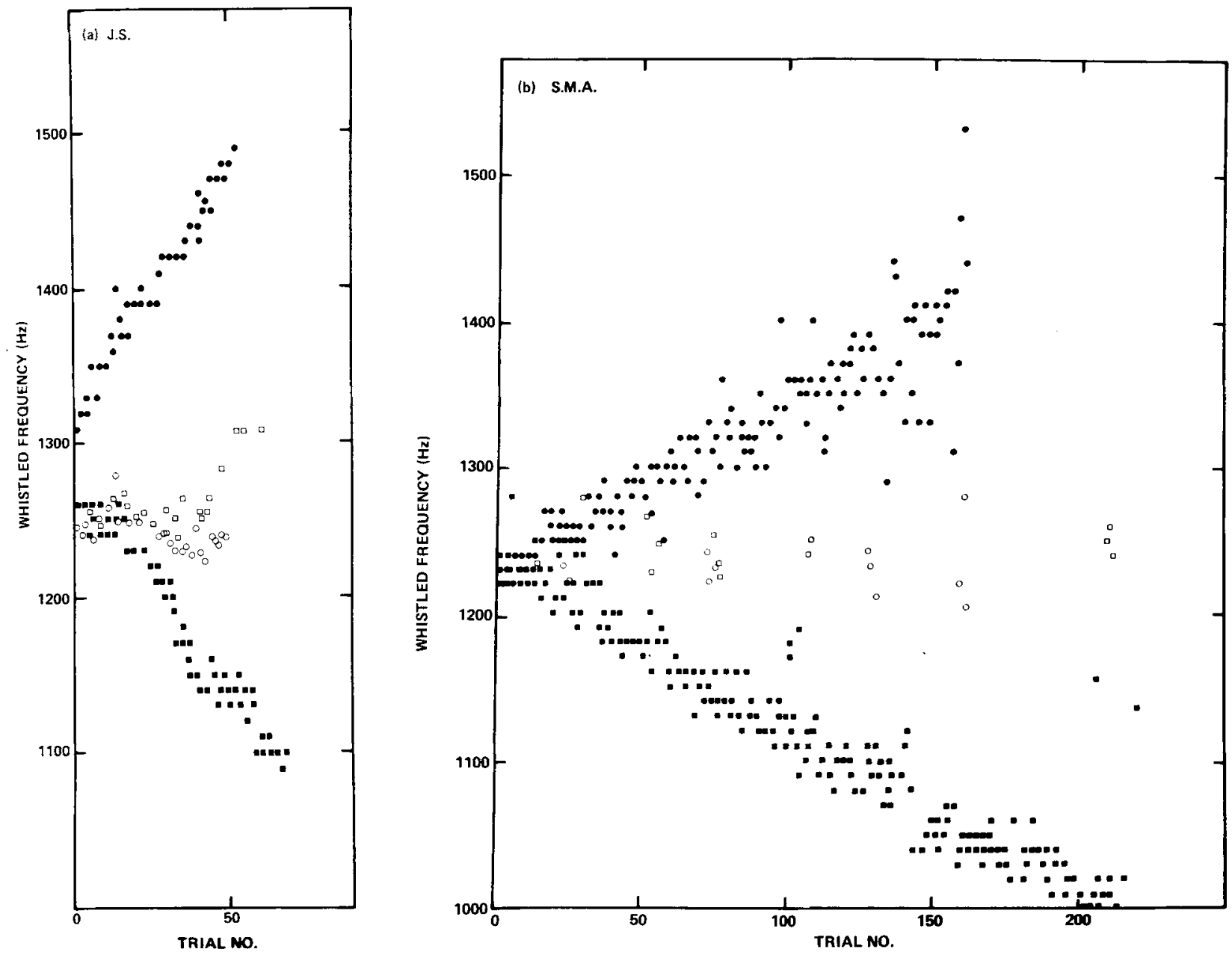

Figure 5. Frequency of whistles produced by subjects as the fedback frequency was gradually reduced to $-250 \mathrm{~Hz}(\bullet)$ or increased to $+250 \mathrm{~Hz}(\bullet)$. Subjects gradually raised or lowered the pitch of their whistles, to hold constant the frequencies which were fed back through the headphones $(\circ \square)$. (a) Experimentally naive subject (J.S.): Electronic frequency shift was automatically increased gradually over a period of about 2 min ( 50 trials). (b) Experienced subject (S.M.A.): Shift increased over a period of about $8 \mathrm{~min}(200$ trials). Results were similar for both subjects, and for other subjects not shown. These results also resemble Figures 2 and 4.

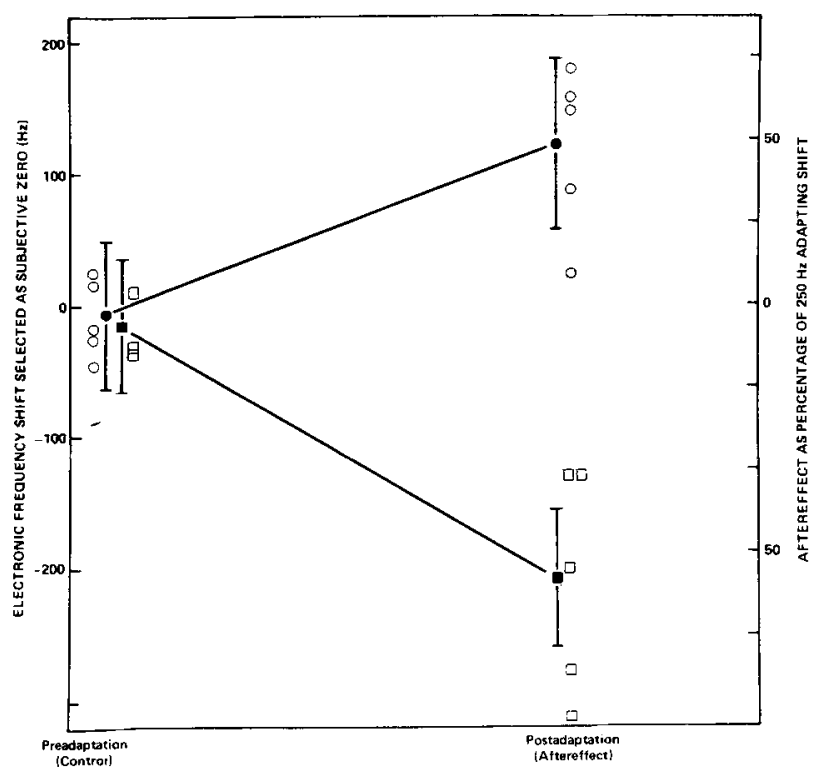

from zero. But after adaptation to a shift of $+250 \mathrm{~Hz}$, the mean setting (postadaptation minus preadaptation) was $+122 \mathrm{~Hz}(49 \%)\left(t_{\mathrm{DEP}}=5.8\right.$, $\mathrm{df}=4$, $\mathrm{p}<.01$, two-tailed test). After adaptation to $-250 \mathrm{~Hz}$, the mean setting was $-192 \mathrm{~Hz}(77 \%)\left(t_{\text {DEP }}=5.6\right.$, $\mathrm{p}<.01$ ). These aftereffects are comparable to the $60 \%$ aftereffects found in the previous experiments, although the method for measuring them was quite different.

Figure 6. Aftereffects measured by asking the subject to reset the electronic frequency shift to subjective zero. Open symbols show individual subjects, filled symbols the mean of five subjects. After adapting, as in Figure 5 , to a frequency shift which gradually increased from zero to $+250 \mathrm{~Hz}(\bullet)$, subjects selected as apparent zero an actual shift of $+122 \mathrm{~Hz}$, or $49 \%$ of the final adapting shift $(p<.01)$. After a final adapting shift of $-250 \mathrm{~Hz}$ $(\square)$, the mean subjective zero setting was $-192 \mathrm{~Hz}$, or $77 \%$ of the final adapting shift $(p<.01)$. These numbers are postadaptation minus preadaptation. Aftereffects are comparable to those shown in Figure 4. 


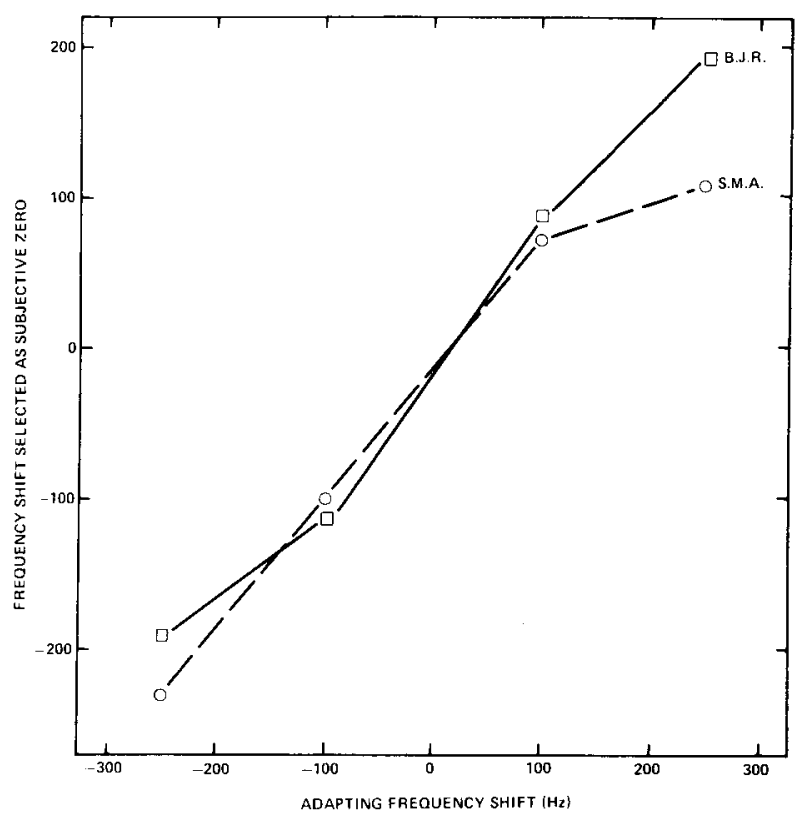

Figure 7. Aftereffects, measured by the reset method, averaged $\pm 92 \mathrm{~Hz}(92 \%)$, following adaptation to $\pm 100 \mathrm{~Hz}$, and $\pm 180 \mathrm{~Hz}$ $(72 \%)$, following adaptation to $\pm 250 \mathrm{~Hz}$. Compare with Figure 3 .

\section{EXPERIMENT 5: RESETTING AFTER ADAPTATION TO \pm 100 AND $\pm 250 \mathrm{~Hz}$}

In our final experiment, two experienced subjects adapted to frequency shifts which were gradually incremented to final values of $-250,-100,+100$, and $+250 \mathrm{~Hz}$ on different runs. The repeated target tone was also eliminated, because we wondered whether it had any undesirable locking effects on the subjects' responses. So, on adapting runs, the target tone was sounded just once at the beginning of the run only. After that, the subject tried to keep whistling at the remembered target frequency about every $2 \mathrm{sec}$ over the ensuing $2 \mathrm{~min}$. Results: We found that suppressing the repeated target tone made no noticeable difference to the subjects' performance. Either with or without a target tone, their whistles gradually shifted up (for a downward frequency shift) or down (for an upward shift), just as before. Aftereffects, measured by the resetting method, averaged $\pm 92 \mathrm{~Hz}(92 \%)$ for shifts of $\pm 100 \mathrm{~Hz}$, and $180 \mathrm{~Hz}$ $(72 \%)$ for shifts of $\pm 250 \mathrm{~Hz}$ (Figure 7).

\section{DISCUSSION}

The control of the vocal tract by means of proprioceptive and auditory feedback can be compared to the control of arm position by means of proprioception and visual feedback. Proprioceptive/motor information from the mouth and tongue may be different from that from the limbs. Some "inflow" information about limb position certainly comes from the receptors in the joint capsules, but there is additional "outflow" information available in the form of an efferent copy or a "sense of effort" (Kelso, 1977). In the case of the extraocular muscles, eye position is almost certainly registered by efferent copy (see Helmholtz, 1866/1963; von Holst, 1954; Howard \& Templeton, 1966; Merton, 1961, 1964), and proprioceptive feedback seems to be minimal or absent (Matin \& Kibler, 1966; Matin, Pearce, Matin, $\&$ Kibler, 1966). It would not be surprising if the system for controlling the vocal tract resembles the eyeball rather than the limbs, because the tongue and eyeball, unlike other parts of the body, are aimed by muscles but are completely lacking in joints. In this paper we do not consider these issues, but have lumped together all motor and proprioceptive cues under the general heading of proprioception. For a discussion of feedback control of human lip muscles, see Bratzlavsky (1979).

Merton (1961) found that the eye and the hand can be aimed in the dark to within an accuracy of about $1 \mathrm{deg}$. This indicates that proprioceptive information from the limbs or the eyeball is calibrated to within about 1 deg. Our Experiment 1 was analogous to this. When auditory feedback was masked off by noise, the voice could still be "aimed" to within about a semitone on the basis of proprioceptive information.

Our Experiments 2 and 3 on adaptation and negative aftereffects from frequency-shifted feedback are auditory analogs of visual adaptation to prisms (for a review, see Howard, 1978). When the visual field is displaced a few degrees to the left by wedge prisms, subjects tend to point past a target, aiming too far to the left. They adapt over a few trials and are soon aiming accurately again. When the prisms are removed, they show a brief negative aftereffect, aiming off to the right. The adaptation probably consists of a change or recalibration in the felt position of the arm, or sometimes of the head's position on the neck (Harris, 1965). In cases of marked cue conflict, vision tends to dominate over touch (Rock \& Harris, 1967).

Howard (1968) suggested that the most effective way to induce prism adaptation was to use variablepower prisms and gradually increase the amount of prism displacement from trial to trial. This leads to a rapid buildup of adaptation, and if the increments are small enough the subject may not be aware that he is adapting, or even realize that any prisms exist.

Our Experiment 3 was an auditory analogue of incremental prism adaptation, with the electronic frequency shift acting as an "auditory prism." The subject whistled, "aiming" at the target frequency. If we put in an unexpected large-frequency shift, the subject's aim was in error and he would hit a wrong note 
and glissando up or down accordingly. By using Howard's incremental technique, we shaped up the subject's responses so that he rapidly adapted and showed a negative aftereffect, making frequency errors in the test condition which were opposite to the artificial electronic shifts. The effects built up during the adaptation period, which lasted only 20$30 \mathrm{sec}$, and outlasted the $30-\mathrm{sec}$ test period.

Experiments 4 and 5 refined this incremental shaping procedure, and used a resetting procedure to measure the aftereffects. This procedure was the auditory analogue of adapting the subject to a prism which gradually increased in refractive power or deviation, and then asking him to reset the deviation of the adjustable prism to zero. It excluded the possibility that the aftereffects were merely conscious corrections made by the subject. The aftereffects here were just as large as those in Experiment 3.

Katz and Lackner (1977) found adaptation to delayed auditory feedback (DAF). DAF disrupts the production of speech, causing an increase in speech duration as well as many articulatory errors. Using both incremental and constant-delay exposure conditions, they found that prolonged exposure to DAF led to adaptive compensation in the form of increased speaking rates. After the DAF was removed, aftereffects were apparent in terms of increased speaking rates.

We have suggested that whistling, and perhaps singing, are controlled by proprioception which gives a coarse calibration of the intended output and by hearing which gives a fine calibration of the actual output. Usually, the finely calibrated feedback overrides proprioception, but the latter provides a backup system which can stand alone in situations where feedback becomes unavailable. Our results show that the feedback system could recalibrate the proprioceptive system within $30 \mathrm{sec}$ or less.

Dual calibration systems in parallel are quite common in engineering practice, and we realized belatedly that we had included one in our experimental setup. Our frequency generator had a precalibrated tuning dial which indicated the intended output frequency to within about five percent. We could use this when we had nothing better, but for more precise work we added a digital frequency meter, which indicated the actual output frequency to within $.1 \%$ or better. At one time we noticed a marked shift or discrepancy between the meter reading and the dial reading. We accepted the meter reading as more reliable and concluded, probably correctly, that the dial had slipped round on its shaft. We adapted to this situation by recalibrating the dial-a simple matter of loosening its set screw and moving it round until its readings again agreed with the meter.

\section{REFERENCES}

Bratzlavsky, M. Feedback control of human lip muscles. Experimental Neurology, 1979, 65, 209-217.

Chase, R. A., Sutton, S., \& Finst, D. Bibliography: Delayed auditory feedback. Journal of Speech and Hearing Research, $1959,2,193.200$.

Harris, C. Perceptual adaptation to inverted, reversed, and displaced vision. Psychological Review, 1965, 72, 419-444.

Hartley-Jones, M. Frequency shifter for howl suppression. Wireless World, 1973, 79, 1453.

Helmholtz, H, von. Handbuch der physiologischen Optik. Leipzig: Voss, 1866. [English translation: $A$ treatise on physiological optics (Vol. 3, 3rd ed., J. P. O. Southall, Ed.). New York: Dover, 1963.]

HoLst, E. von. Relations between the central nervous system and the peripheral organs. British Journal of Animal Behaviour, 1954, 2, 89-94.

How ARD, I. P. Displacing the optical array. In S. J. Freeman (Ed.), The neuropsychology of spatially oriented behavior. Homewood, Ill: Dorsey Press, 1968.

How ARD, I. P. Effects of exposure to spatially distorted stimuli. In R. D. Walk \& H. L. Pick (Eds.), Perception and experience. New York: Plenum, 1978.

Howard, I. P., \& Anstis, T. Muscular and joint-receptor components in postural persistence. Journal of Experimental Psychology, 1974, 103, 167-170.

How ARD, I. P., \& Templeton, W. B. Human spatial orientation. London: Wiley, 1966.

Katz, D. I., \& Lackner, J. R. Adaptation to delayed auditory feedback. Perception \& Psychophysics, 1977, 22, 476-486.

KeLso, J. A. S. Motor control mechanisms underlying human movement reproduction. Journal of Experimental Psychology: Human Perception and Performance, 1977, 3, 529-543.

Matin, L., \& KibleR, G. Acuity of visual perception of direction in the dark for various positions of the eye in the orbit. Perception and Motor Skills, 1966, 22, 407-420.

Matin, L., Pearce, D., Matin, E., \& Kibler, G. Visual perception of direction in the dark: Roles of local signs, eye movements and ocular proprioception. Vision Research, 1966, 6, 453-469.

Merton, P. A. The accuracy of directing the eyes and the hand in the dark. Journal of Physiology, 1961, 156, 555-557.

Merton, P. A. Human position sense and sense of effort. Homeostasis and feedback mechanisms. 18th Symposium of the Society of Experimental Biology. Cambridge, England: Cambridge University Press, 1964.

Rock, I., \& Harris, C. Vision and touch. Scientific American, Offprint 507, May 1967.

\section{NOTE}

1. Surrey Electronics, The Old Forge, Lucks Green, near Cranleigh, Surrey, England.

(Received for publication February 27, 1979; revision accepted October $14,1979$. 